\title{
Índice de Segregación Espacial y Socioeconómico (ISES) en las comunas de Santiago de Cali*
}



Resumen La segregación socioeconómica del espacio urbano tiene relaciones complejas según la condición étnica, etaria, educativa, cultural u origen migratorio, entre otras. En este contexto, se realizó un estudio espacial en Cali, mediante un análisis multivariado, con aplicación del escalograma de Guttman. Los resultados por comunas muestran que mientras la ciudad se extiende hacia las periferias, la segregación espacial aumenta hacia las laderas en el occidente y las márgenes del río Cauca en el oriente, lo que coincide con la localización de grupos étnicos o en condición de pobreza, a diferencia de grupos socioeconómicos privilegiados, que tienden a concentrarse en el norte y sur de la ciudad.

Palabras clave Ambiente; Cali (Colombia); globalización; población; segregación espacial y socioeconómica

Palabras clave Segregación; globalización; población; vida cotidiana - Cali (Colombia) descriptor 


\section{Socioeconomic and Spatial Segregation Index (ISES by its initials in Spanish) in the Municipalities of Santiago de Cali}

Abstract Socioeconomic segregation of urban space has complex relationships according to ethnicity, age, education, culture or immigration status, among others. In this context, a spatial study was conducted in Cali, by multivariate analysis, applying the Guttman scalogram. The results by municipalities show that as the city extends to the periphery, spatial segregation increases towards the slopes in the west and the banks of the Cauca River in the east, which coincides with the location of ethnic groups or people in poverty, unlike privileged socioeconomic groups, which tend to be concentrated in the north and south of the city.

Key words Environment; Cali (Colombia); globalization; population; spatial and socioeconomic segregation

Key words Segregation; globalization; population; everyday life - Cali (Colombia) plus

\section{Índice de Segregação Espacial e Socioeconómico (ISES) nas comunas de Santiago de Cali}

Resumo A segregação socioeconómica do espaço urbano tem relações complexas de acordo à condição étnica, etária, educativa, cultural e a origem migratória, entre outras. Neste contexto, realizou-se estudo espacial em Cali, através de análise multivariada, com aplicação do escalograma de Guttman. Os resultados por comunas mostram que enquanto a cidade estende-se para as periferias, a segregaçáo espacial aumenta para as ladeiras no oeste e as margens do rio Cauca no leste, o que coincide com a localização de grupos étnicos ou em condição de pobreza, ao contrário de grupos socioeconómicos privilegiados, que visam a se concentrar no norte e sul da cidade.

Palavras chave Ambiente; Cali (Colônia); globalização; população; segregação espacial e socioeconómica

Palavras chave Segregação urbana; globalização; população; vida cotidiana - Cali (Colombia) descritor 


\section{Introducción}

La globalización es una creciente comunicación e interdependencia entre los países del mundo, que unifica sus mercados, sociedades y culturas por medio de una serie de transformaciones sociales, económicas y políticas que, sumadas al desarrollo digital, han generado la concentración de modelos emergentes de gestión financiera e internacionalización del capital en torno a ciudades mundiales hacia donde se mueve el mercado de servicios, finanzas e innovación, que luego se difunde al resto de ciudades; estas han adoptado diferentes estrategias para hacer frente a las nuevas condiciones económicas, cuyo éxito depende de la capacidad de recibir inversión extranjera y de exportar (Sassen, 1991). Autores como Brenner (2001), Cepal (2002), Stallings (2001) y Taylor (2003) analizan los retos de la globalización.

Este proceso ocurre a costa de una gran cantidad de fracturas, por lo que se considera que esta transformación va acompañada de otra denominada fragmentación (Celemín, 2012; Uribe, 1998). Este esquema global, además de cambiar el tejido económico, social y ambiental de las grandes áreas urbanas, también reforma su estructura espacial (Sachs-Jeantet, 1995, p. 7); su marca queda registrada en la territorialidad de las ciudades al acentuar sus disparidades internas y favorecer algunos sectores, mientras otros quedan marginados (Celemín, 2012).

Esta diferenciación espacial ha existido siempre; sin embargo, en la actualidad se observa con mayor nitidez y amplitud en los espacios de ciudades en desarrollo. Se presenta una clara distinción entre los que tienen mucho y los que no tienen y se exhibe un espacio más fragmentado y segregado. En este marco, Alvarado, Vieyra y Hernández (2008) y Janoschka (2002) mencionan que existe una fragmentación de los usos del suelo como nuevas formas de la expresión espacial de las ciudades de América Latina a finales del siglo XX y principios del XXI. A la vez, crecen los espacios destinados a la población de bajos ingresos, que se encuentran aislados por grupos de población de nivel socioeconómico mayor; en esta región, la población urbana representa más de $80 \%$ de la población total, crece junto a la pobreza y aumenta la producción social de riesgo por el uso de áreas de amenaza (Meyer y Bahr, 2001; De Mattos e Hidalgo, 2007).

Lévy y Brun (2002) definen la segregación como "una forma de distribución desigual de grupos de población en el territorio" (p. 147) que se manifiesta de diferentes formas: la proximidad física entre espacios residenciales de los diferentes grupos sociales (White, 1983), por homogeneidad social de las subdivisiones territoriales en las que se estructura una ciudad (Sabatini, 1999) o por la concentración de grupos sociales en áreas específicas de la misma (Rodríguez, 2001; Massey, White y Phua, 1996). Sabatini, Cáceres y Cerda (2001) la redefinen como: "El grado de proximidad espacial o de aglomeración territorial de las familias pertenecientes a un mismo grupo social, sea que éste se defina en términos étnicos, etarios, de preferencias religiosas o socioeconómicos, entre otras posibilidades" (p. 10). 
Este fenómeno no ha sido ajeno a las ciudades colombianas. En el caso de Cali, estudios realizados por Vásquez (2001) indican que, hacia el último período del siglo XX, la urbe consiguió modificar la tendencia de su desarrollo hacia el sector terciario de la economía y presentarse como una ciudad dispuesta a participar en la globalización del sistema económico, aunque también quedó condicionada por las desigualdades y los sobresaltos propios de ese nuevo mundo económico en el que decide insertarse.

Como resultado de esta nueva dinámica, Cali presenta una tendencia negativa de sustentabilidad, los problemas económicos han aumentado en la medida que han disminuido los ingresos de la población y los problemas ambientales están directamente relacionados con la situación socioeconómica de la población. Hay una correspondencia entre los indicadores y las consecuencias de estas interacciones que podrían resumirse en una mayor segregación social y grandes diferencias en la dualidad urbana de la ciudad formal y la informal (Observatorio Social [OS], 2007) con lo que se generan brechas sociales de diverso orden: generacionales, étnicas, socioeconómicas y políticas; por ello la armonía y el respeto se han convertido en desafíos lejanos (Observatorio Internacional de la Democracia Participativa [OIDP], 2008).

Desde este enfoque, el análisis de segregación espacial y socioeconómico es una de las cuestiones de mayor importancia al momento de estudiar la relación sociedad-espacio en el ámbito urbano, al considerarse el importante efecto de la globalización actual, ya que, como señala Ainstein (1998), las ciudades se desarrollan en un creciente contexto de integración mundial, pero no como estructuras integradas, pues en su interior se producen las mayores asimetrías (Buzai, Baxendale, Rodríguez y Escanes, 2003). Este artículo presenta los resultados del análisis de se- gregación espacial y socioeconómica en la ciudad de Cali, según variables socioeconómicas, demográficas, étnicas y ambientales del año 2011, obtenidas del Departamento Administrativo de Planeación, la Alcaldía de Santiago de Cali, el análisis estadístico del Sisbén, el Plan Operativo Anual de Inversiones de Cali (Ejecución vigencia 2011) y la ejecución presupuestal de la inversión por comuna a 31 de diciembre de 2011.

El propósito es evidenciar la necesidad de un cambio en la tendencia negativa a la sustentabilidad, provocada por los impactos de la segregación espacial y poblacional, para propiciar una nueva perspectiva que motive a los sectores de la vida municipal, urbana y metropolitana a la acción conjunta del escenario futuro de una Cali sostenible, que articule lo social, económico, ambiental, técnico y político en un proceso de planificación integral.

\section{Globalización y metropolización en Cali: evidencias de segregación espacial y socioeconómica}

El proceso de metropolización se evidencia en aquellas ciudades que han asumido de forma espontánea o planificada la responsabilidad de organizar un espacio a su alrededor. Al privilegiarse la localización de servicios y equipamientos en un determinado núcleo urbano, se propician flujos de personas y mercancías que se intensifican con el paso del tiempo. La naturaleza misma de este fenómeno hace que se sobreponga a las divisiones político-administrativas territoriales y las desborde. Cuando esto ocurre, las problemáticas, que antes eran de impacto municipal, ahora involucran más de un municipio e incrementan la complejidad de las soluciones; por tal motivo, entender este fenómeno y caracterizarlo es de vital importancia para cualquier acción que pretenda orientar su planificación (Martínez, 2005). 
Por su dinámica y elevada tendencia a la concentración de la oferta de bienes y servicios, Cali se convirtió en un municipio núcleo o metrópoli; durante el período de 1933 a 1955, se inició un proceso de desarrollo promovido por la industrialización acelerada y el profundo impacto de la migración. En este lapso de apogeo industrial se identifican varios fenómenos de gran importancia: cambios tecnológicos, aceleración de la inmigración, crecimiento urbano, intensificación de la invasión de tierras para uso residencial y despliegue de la ciudad hacia el oriente. Estos fenómenos constituyeron fuerzas en expansión constreñidas por una estructura física tradicional que no favorecía el desarrollo económico y social, lo que desencadenó una aguda crisis social y urbanística caracterizada por la proliferación de intensas luchas populares por la tierra y los ejidos y por una fuerte agitación sindical y huelguística (Vásquez, 2001).

El último período del siglo XX se definió por la desaceleración de la dinámica económica, la ocupación de nuevos espacios, la transición demográfica y una exclusión socioespacial producto de la misma. Cali, ahora una ciudad metropolitana, entró en un momento histórico señalado por la crisis económica, el desempleo, la reproducción del narcotráfico, las crecientes brechas sociales y la agudización de la segregación socioespacial (Vásquez, 2001). Evidencias de este crecimiento se consignan en un estudio realizado por la Universidad San Buenaventura (2011), en el que los autores analizan el crecimiento de las ciudades del entorno metropolitano de Cali en tres períodos: 1961, 1998 y 2007. La Tabla 1 muestra las tasas de crecimiento.

La consecuencia es un tipo de ciudad que, en rasgos generales, aparece fragmentada entre un sector social de la población con acceso al consumo y los servicios y otro sumido en la pobreza y a pocos pasos de la exclusión social (Clichevsky, 2002, p. 12). Existen diferencias marcadas en las condiciones ambientales de los habitantes del oriente de Cali y quienes habitan las comunas "privilegiadas" de la ciudad. De acuerdo con el Plan de Ordenamiento Territorial, el índice de vegetación por habitante muestra que las comunas 17,19 y 22 presentan los mejores escenarios, con zonas verdes mayores a $10 \mathrm{~m}^{2}$ por habitante y en ocasiones cercanas a $30 \mathrm{~m}^{2}$; por el contrario, las comunas del oriente (distrito de Aguablanca) no alcanzan valores superiores a $2 \mathrm{~m}^{2}$, lo que marca grandes desigualdades en cuanto a calidad ambiental (Escobar-Morales, 2012).

La contribución resultante del análisis económico y geográfico consiste en reconocer que la

Tabla 1.

Tasa de crecimiento de área construida en los municipios del área de influencia metropolitana de Cali

\begin{tabular}{|c|c|c|c|c|c|}
\hline \multirow{2}{*}{ Municipio } & Construcción 1961 & Construcción 1998 & Construcción 2007 & $\begin{array}{c}\text { Tasa de crecimiento } \\
1961-1998\end{array}$ & $\begin{array}{c}\text { Tasa de crecimiento } \\
1998-2007\end{array}$ \\
\hline & \multicolumn{3}{|c|}{ Área (Has.) } & \multicolumn{2}{|c|}{ Porcentaje } \\
\hline Cali & 2296,9 & 9487,5 & 11414,6 & $313 \%$ & $20 \%$ \\
\hline Candelaria & 137,8 & 442,1 & 510,2 & $221 \%$ & $15 \%$ \\
\hline Jamundí & 66,8 & 503,7 & 769,7 & $654 \%$ & $53 \%$ \\
\hline Palmira & 377,8 & 1715,2 & 2299,9 & $354 \%$ & $34 \%$ \\
\hline Yumbo & 143,5 & 902,7 & 1283,8 & $529 \%$ & $42 \%$ \\
\hline
\end{tabular}

Fuente: Universidad de San Buenaventura, seccional Cali (2011) 
localización en el espacio tiene mucha importancia en las condiciones socioeconómicas y de vida de las personas. En tal sentido, las disparidades territoriales son un reflejo de las inequidades de la población dentro de una sociedad definida temporal y espacialmente. El proceso contemporáneo de fragmentación social y territorial de las condiciones de vida de la población se manifiesta, entonces, como un nuevo mecanismo de exclusión social y segregación espacial, que transforma de modo sustancial la dinámica y la configuración de un diverso mosaico de subespacios (Celemín, 2012).

\section{Agentes causales de segregación}

El estudio de la segregación espacial tiene una larga trayectoria académica, sobre todo a partir de los trabajos de la Escuela de Chicago a principios del siglo XX. El análisis puede abordarse desde dos grandes aproximaciones: por una parte, desde la perspectiva del inmigrante, es decir, cómo influye el hecho de estar o no segregado en el proceso de integración en la sociedad receptora y, por otra parte, desde la perspectiva del territorio y de la sociedad de acogida, o sea, cómo influye la llegada de la inmigración en la transformación socioespacial de los barrios. En los últimos años, diferentes trabajos publicados dejan entrever el creciente interés sobre el objeto de estudio (Fullaondo, 2008; Bayona, 2007; Checa y Arjona, 2006).

Arriagada y Rodríguez (2003) examinan la segregación residencial en áreas metropolitanas de América Latina, su magnitud, sus características, su evolución y sus implicaciones; identifican un patrón de concentración territorial de los grupos de mayor ingreso en zonas integradas y bien conectadas con la ciudad, mientras los de menores ingresos tienden a distribuirse en la periferia suburbana. La migración intrametropolitana es selectiva y conlleva a la segregación residencial socioeconómica, aunque esto podría estar cambiando en algunas ciudades donde la ampliación de la infraestructura ha facilitado la reubicación de grupos de la élite. Aunque la segregación residencial favorece la reproducción de las desigualdades socioeconómicas y dificulta los esfuerzos descentralizadores y de gobernabilidad urbana, en la región hay pocas intervenciones. Se destacan varios estudios: en Chile, Armijo (2000), Arriagada y Simioni (2001) y Sabatini, (1999); en Colombia, Barbary (2001) y en Uruguay, Katzman, Retamoso y Corbo (2003).

A continuación se muestra un esquema general del estudio de la segregación espacial, con el objeto de dar una visión general y transversal de las variables y los factores que se han de tener en consideración a la hora de abordar esta temática. Cuando un país se convierte en receptor de inmigración y los flujos hacia el mismo se vuelven intensos, el fenómeno migratorio tiende a convertirse en un factor de transformación social. Sin embargo, las ciudades no son homogéneas en su estructura urbana ni en su estructura social; se caracterizan, más bien, por tener una determinada morfología social y urbana (Martínez y López, 2002; Leal, 2007). En este sentido, tanto la jerarquía residencial como el mercado inmobiliario son factores que inciden en la distribución de los inmigrantes en la ciudad (White, 1995, p. 68). Las ciudades se caracterizan por la existencia de una diferenciación de la vivienda en términos de calidades, superficies, valor, etc. Esta jerarquía residencial origina la primera delimitación territorial de las zonas en las que la vivienda es más accesible para la población con menos recursos.

Las Figuras 1 y 2 muestran las principales carencias de los hogares respecto a ciertos bienes y servicios básicos para subsistir. En la Figura 1 se presenta el número de hogares con viviendas inadecuadas (viviendas móviles, refugios, puentes, paredes de telas o desechos, piso de tierra, etc.) y 
Figura 1.

Número de hogares con vivienda inadecuada $\left(\mathrm{Hog} / \mathrm{Km}^{2}\right)$

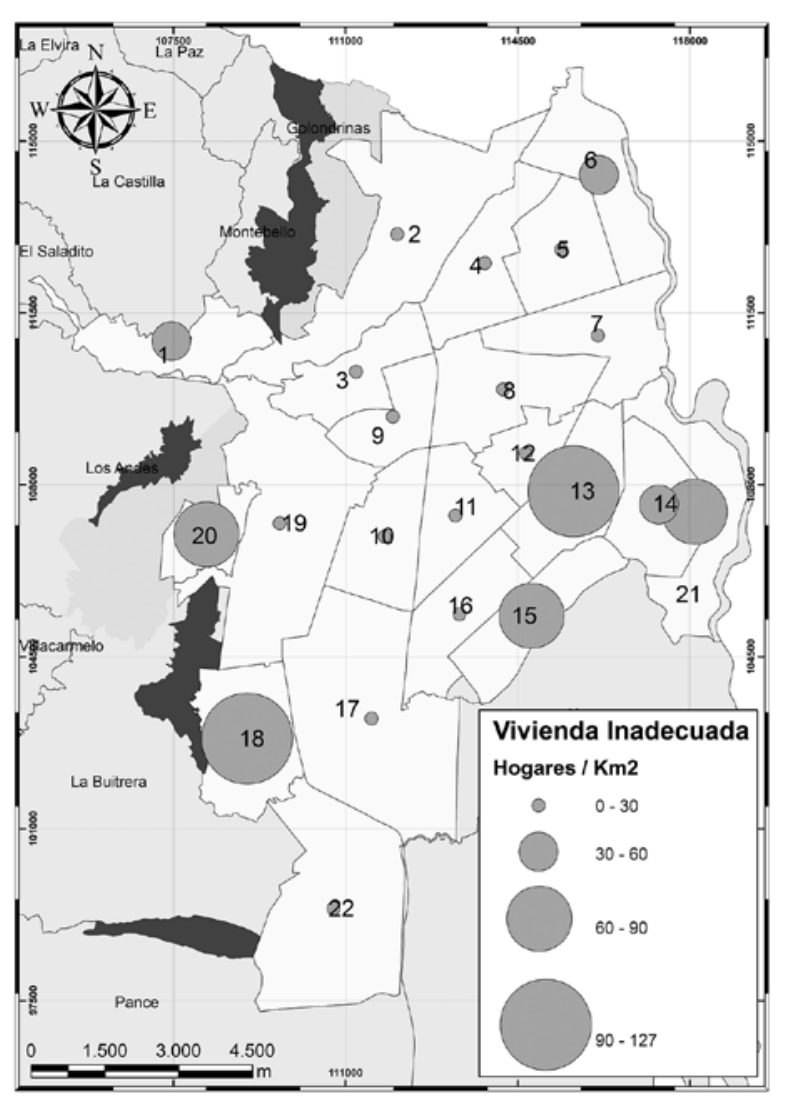

Fuente: elaboracion propia con datos del Departamento Administrativo de Planeación (2011)

la Figura 2 indica los hogares con hacinamiento crítico (hogares con tres personas por cuarto); los dos mapas señalan que las comunas donde existe mayor cantidad de hogares con carencia están localizadas en las áreas periféricas de la ciudad, sobre todo en las comunas $6,13,14,15,18$ y 21 y en las comunas de la zona de ladera al occidente de Cali (1 y 20). Por el contrario, las comunas con los estratos socioeconómicos más altos (17, 19 y 22) indican una cantidad mínima de hogares con algunos de los problemas mencionados.

$\mathrm{Si}$ a los dos indicadores se les suman condiciones como servicios inadecuados, inasistencia escolar y dependencia económica, se puede obtener el índice de Necesidades Básicas Insatisfechas (NBI); este determina que si el hogar cuenta
Figura 2.

Número de hogares con hacinamiento crítico $\left(\mathrm{Hog} / \mathrm{Km}^{2}\right)$

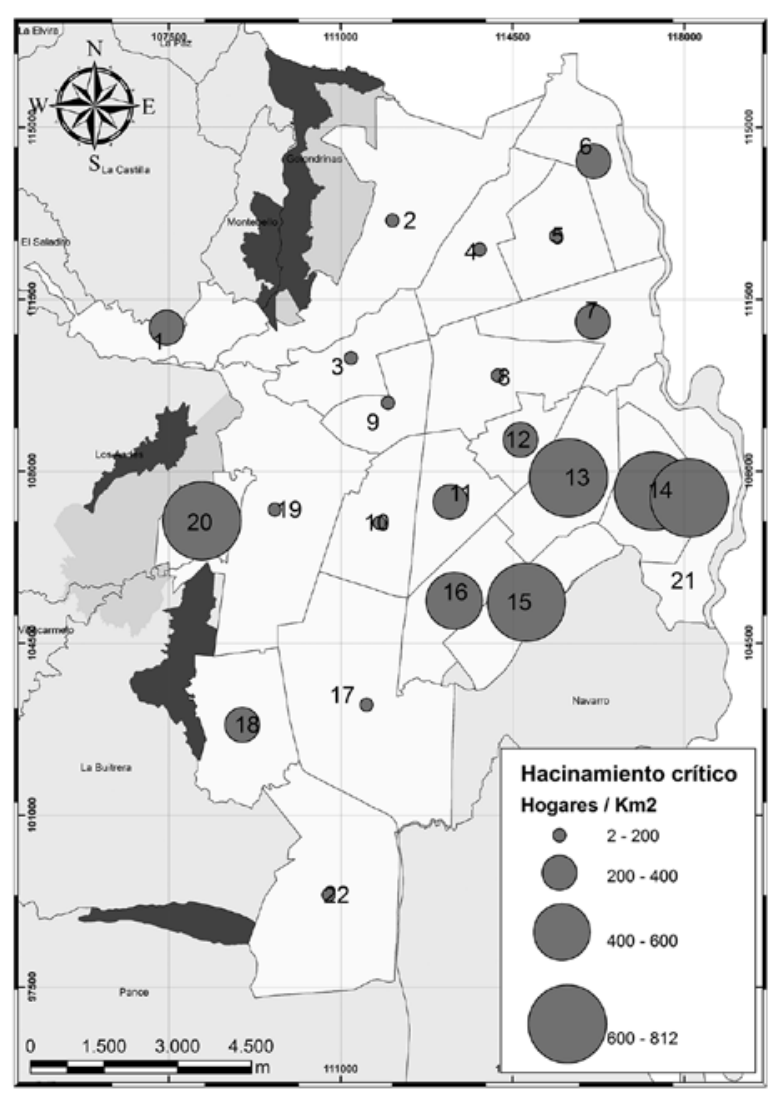

con al menos una de ellas, tiene NBI, pero si tiene dos o más carencias, está en condición de miseria. La Figura 3 indica que las comunas que presentan el mayor número de hogares en condición de miseria se encuentran al oriente de Cali, en las comunas $6,13,14,15$ y 21 y las comunas de la ladera $(1,18$ y 20$)$.

En lo que respecta a las causas de la segregación, Bayona (2007) afirma que, desde el punto de vista geográfico, existen tres factores inductores: motivos socioeconómicos, motivos demográficos y motivos étnicos. El factor socioeconómico se relaciona con la condición de clase desfavorecida de la inmigración, lo que provoca que la diferenciación socioeconómica de la ciudad sea un importante factor de segregación residencial. 
Figura 3.

Hogares en condición de miseria

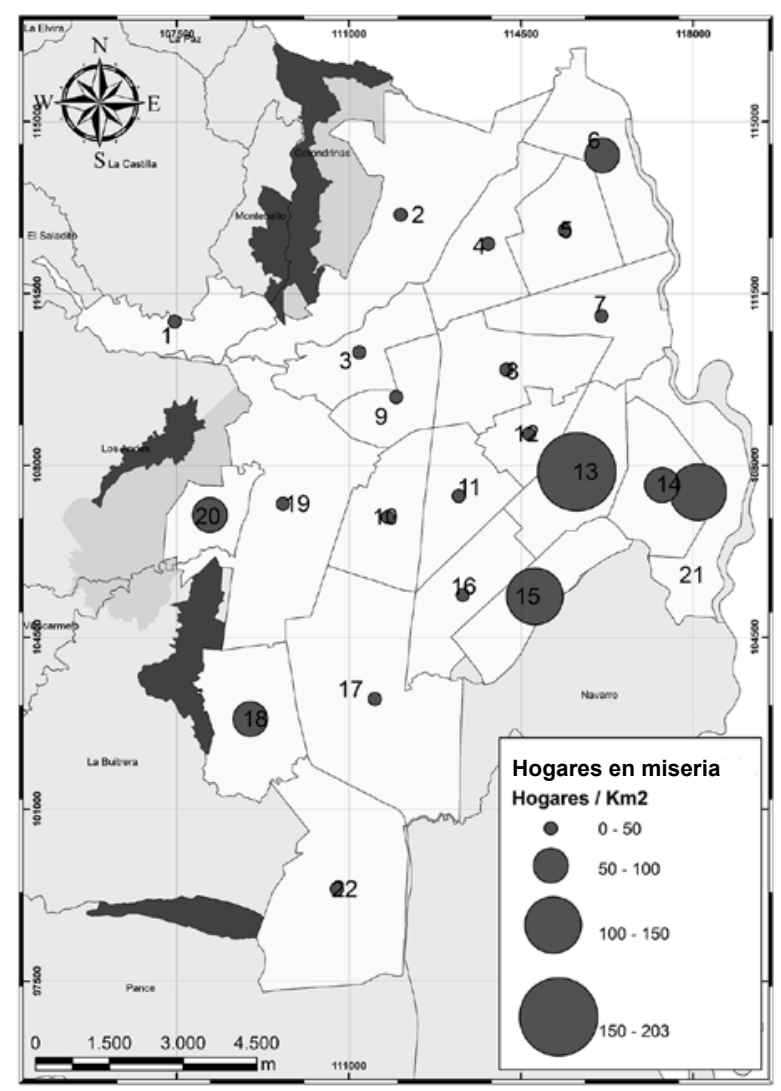

Fuente: elaboracion propia con datos del Departamento Administrativo de Planeación (2011)

El factor demográfico apunta a la estructura del hogar condicionado por el proceso migratorio como elemento de diferenciación residencial entre los propios inmigrantes. Por último, el motivo étnico se refiere a la distinción social del espacio basado en la etnicidad, que puede responder a procesos endógenos de agrupación o a situaciones de discriminación (Fullaondo, 2007).

Como se puede observar en las Figuras 4 a 7, los estratos socioeconómicos, la cobertura de servicios de alcantarillado, acueducto y energía y el avalúo de los predios de cada comuna tienen una relación inversamente proporcional al contrastarlos con las comunas en donde se localiza la mayor cantidad de comunidades étnicas (afrocolombianos, indígenas, rom y raizales); se identifica que las comunas en las que se ubica la mayoría de las comunidades étnicas $(6,11,13,14,15 \mathrm{y}$ 21) son también las comunas con los estratos socioeconómicos más bajos, cuyos predios tienen los menores precios y la cobertura de los servicios es la más baja en toda la ciudad, a diferencia de las comunas con estratos altos (2, 17 y 22), en las que se cuenta con la mayor cobertura de servicios, la mejor valoración de los predios y un porcentaje menor de comunidades étnicas. Estos resultados muestran, una vez más, cierta segregación social y espacial, condicionada por las variables descritas.

Esta dinámica origina un modelo de desarrollo urbano característico de la era de la globalización, caracterizado por una urbanización difusa y discontinua mediante productos urbanos constitutivos de enclaves o parques temáticos mercantilizados (de ocio, comerciales, empresariales, etc.) y áreas degradadas o marginales. Así, la tendencia dominante es la del urbanismo de la privatización, de la distinción y del miedo y esto se expresa en todas las escalas: latinoamericana, nacional, regional y local (Juárez, 2006, p. 3).

Para Cabrales (2002), las principales causas que afectan la cohesión social en las ciudades de América Latina son la polarización de la riqueza, la pauperización de la población, la represión política, las prácticas mafiosas y el tráfico de droga. Los resultados son un esquema de segregación latinoamericano distinguido por concentración espacial de los grupos altos y medios ascendentes en el extremo de la ciudad, con vértice en el centro histórico, llamados "barrios de alta renta"; la conformación de amplias áreas de alojamiento de los grupos pobres, sobre todo en la periferia lejana y mal servida, pero también en sectores deteriorados cercanos al Centro, así como la significativa diversidad social de los "barrios de alta renta”, en donde, además de la virtual totalidad de las élites, viven grupos medios y bajos (Sabatini 2003). 
Figura 4.

Estratos socioeconómicos



Figura 6.

Avalúo de predios (en millones de pesos)

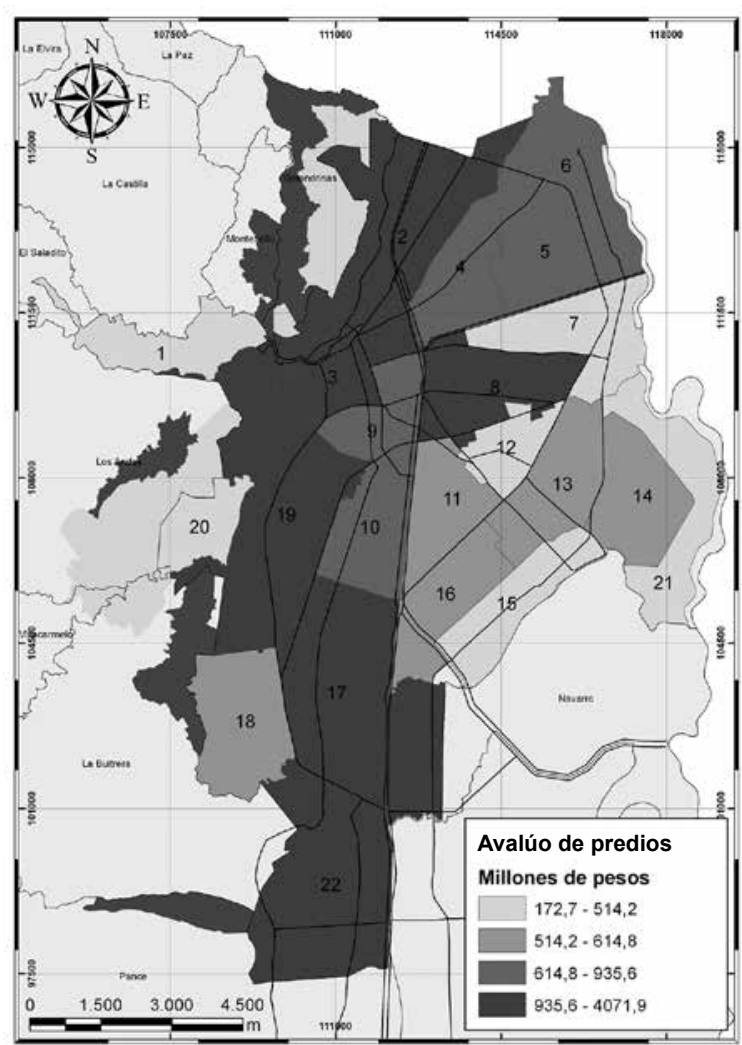

Figura 5.

Número de habitantes de comunidades étnicas por comuna

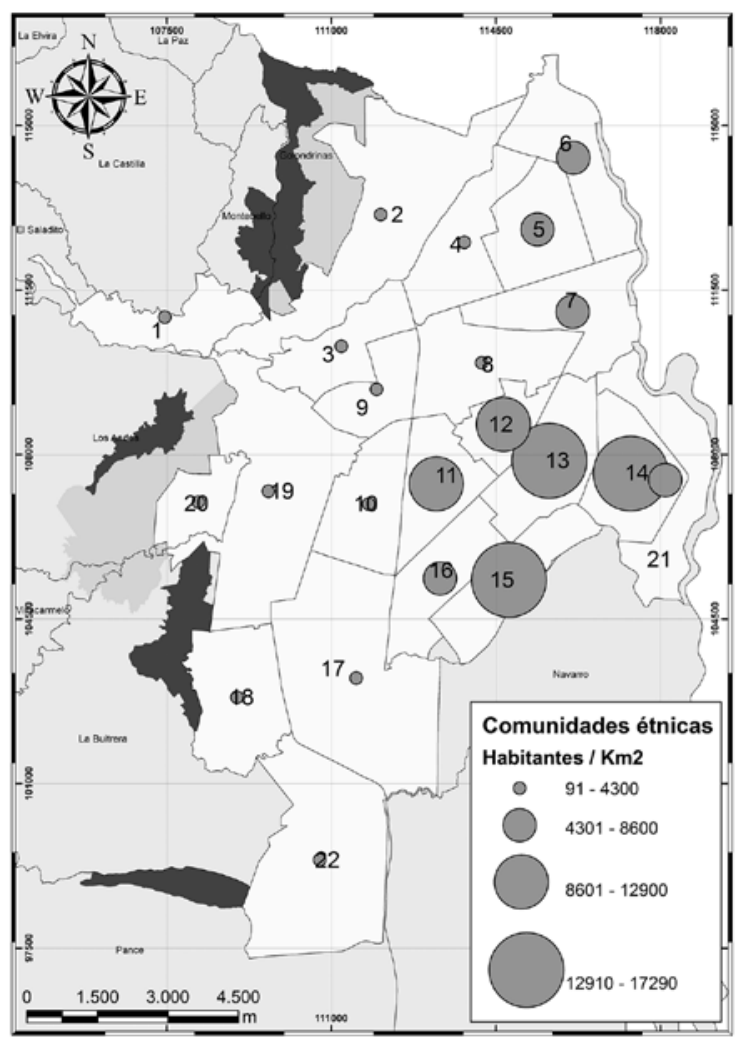

Figura 7.

Cobertura de servicios de alcantarillado, acueducto y energía

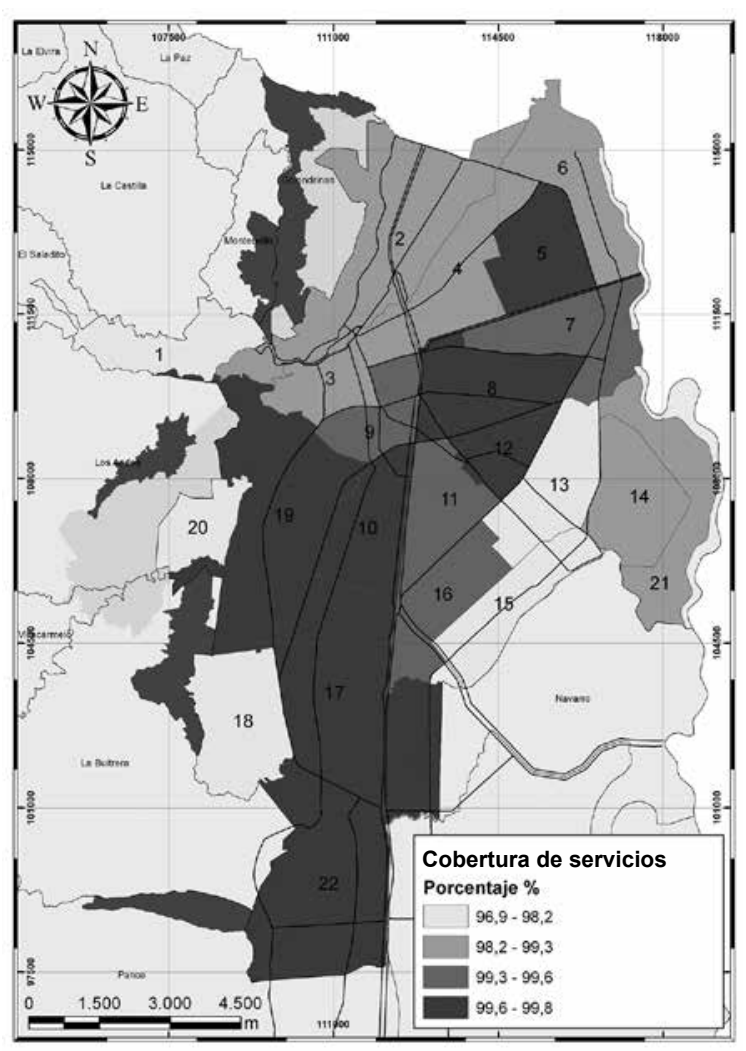

Fuente: elaboracion propia con datos de Escobar-Morales (2012) 
A partir de lo anterior, se construyó un índice de segregación espacial, que permite simplificar los datos y las variables que explican este fenómeno para estructurarlos en una información más elaborada que sintetice la interpretación del problema en cuestión.

\section{Metodología}

De acuerdo con estudios realizados por Polanco (2006), TAU Consultora Ambiental (2000) y Escobar (2008), pasar de la compilación de indicadores simples a indicadores sintéticos es un imperativo para simplificar los datos y estructurarlos en información más elaborada, puesto que los indicadores simples hacen mención a una estadística no muy elaborada, adquirida directamente de la realidad. Los índices son medidas adimensionales que resultan de combinar varios indicadores simples, mediante un sistema de ponderación que jerarquiza los componentes (Castro, 2002, p. 194). La información obtenida posee un significado más amplio que el directamente asociado con cada indicador, por lo cual se precisa una definición clara de ellos, de fácil interpretación, respuesta a cambios y representatividad en el marco de la investigación (Loaiza y Buitrago, 2009).

La importancia de un indicador síntesis radica en sus tres funciones básicas: la primera es la simplificación, es decir, los indicadores son representaciones empíricas de la realidad en las que se reduce el número de componentes; la segunda es la cuantificación, pues mide cuantitativamente (al menos establece una escala) de la problemática estudiada. Por último está la comunicación ya que el indicador debe ser utilizado para transmitir la información referente al objeto de estudio (Organización para la Cooperación y el Desarrollo Económico [OCDE], 1997 citado en Castro, 2002, p. 195).
A partir de las variables que potencian o restringen la segregación espacial y socioeconómica en Cali, se establece el ISES por comuna, con el fin de identificar cuáles son las que presentan el mayor índice asociado con este fenómeno. De acuerdo con la Ley 152 de 1994 y la 388 de 1997 se escogió la comuna, puesto que es la unidad mínima de gestión pública de la ciudad, donde actúan directamente las instituciones y entidades locales, además de que concentran los intereses de la población.

Para el cálculo del ISES se utilizó el escalograma de Guttman, también conocido como el análisis del escalograma. Según Rondinelli:

Este es un método de análisis multivariado que permite determinar la ponderación, correlación y jerarquía de variables objeto de análisis, sin restricciones y de acuerdo a la disponibilidad de la información [...] que permite agrupar diversas modalidades de variables (cualitativas y cuantitativas), ordenar y clasificar series estadísticas, así como definir la frecuencia de los valores de un carácter (1988, p. 161).

Los procedimientos para calcular el ISES fueron:

1. Selección de las variables que permiten definir el fenómeno de segregación para cada comuna; fueron contextualizadas y justificadas en el anterior apartado y se presentan en la Tabla 2.

2. Aplicación de clústeres para la selección de los rangos de variables: en esta etapa se determinaron las categorías en las que serán manejados los datos de las variables de cada comuna. Esta técnica estadística también se conoce como el análisis de conglomerados, cuyo propósito es dividir un conjunto de datos en grupos, de manera que los perfiles de los datos en un mismo grupo sean muy similares entre sí. A partir del análisis de la amplitud de los datos de cada variable, se determinó que el número de clases adecuado era cuatro para todas las variables utilizadas en el cálculo del índice de segregación. 
Tabla 2.

Variables para el análisis de segregación espacial y socioeconómica

\section{Tipo de variable Variable}

\begin{tabular}{|c|c|}
\hline \multirow{2}{*}{ Demográfica } & Comunidades étnicas \\
\hline & Analfabetismo \\
\hline \multirow{8}{*}{ Socioeconómica } & $\begin{array}{l}\text { Cobertura de servicios (acueducto, } \\
\text { alcantarillado y energía) }\end{array}$ \\
\hline & Densidad de población \\
\hline & Estrato socioeconómico \\
\hline & Avaluó de predios \\
\hline & $\mathrm{N}^{0}$ de hogares con vivienda inadecuada \\
\hline & $\mathrm{N}^{0}$ de hogares con inasistencia escolar \\
\hline & $\mathrm{N}^{0}$ de hogares con hacinamiento crítico \\
\hline & $\mathrm{N}^{0}$ de hogares bajo línea de miseria \\
\hline \multirow{3}{*}{$\begin{array}{l}\text { Ambiente, recursos } \\
\text { naturales e inversión }\end{array}$} & Espacio verde por habitante \\
\hline & Inversión del municipio \\
\hline & Inversión en gestión y ambiente \\
\hline
\end{tabular}

Fuente: elaboracion propia

3. Diseño y diligenciamiento del escalograma de Guttman: en la última etapa se procedió a calificar cada variable por cada comuna, mediante el escalograma de Guttman. Este método se utilizó para armonizar variables altamente heterogéneas en un único valor que describiera la situación de segregación en cada unidad. La escala de Guttman es unidimensional y acumulativa; esto implica que los ítems se pueden ordenar por su grado de dificultad y, por ello, la comuna que cumple con un ítem difícil, tendrá siempre una respuesta afirmativa a un ítem menos difícil. Por lo tanto, la información de la posición de la última respuesta positiva permitirá predecir todas sus respuestas a los ítems. El rango del índice es de cero a uno, en donde uno representa el grado perfecto de acumulación. Una vez determinado el número de rangos para las variables, se elaboró la estructura de la matriz del escalograma de Guttman (Tabla 3).

El escalograma de Guttman está compuesto por una primera columna en la que se ubicaron las 22 comunas de Cali. Seguido de estas aparecen cuatro columnas por cada variable establecida para el análisis (52 en total), que representan los rangos determinados en la fase anterior mediante análisis clúster. En las 52 columnas se calificó la ponderación del dato de cada comuna para las 13 variables establecidas. La Tabla 3 muestra la estructura del escalograma de $\mathrm{Gu}$ ttman con los resultados obtenidos para el índice de segregación.

Al final de cada columna se incluyó la frecuencia de la respectiva clase. Seguido a este dato, para considerar la exclusividad de las comunas por clase, se calculó el resultado de dividir el número total de comunas de la ciudad entre la frecuencia de clase de cada variable, valor que luego se reemplazó en la misma tabla por la ponderación de clase para cada comuna. La sumatoria de estos valores presentes en cada fila representa el dato síntesis del número total de variables y corresponde al ISES para las comunas de Santiago de Cali.

\section{Análisis de resultados}

La distribución espacial del ISES indica zonas que pueden ser determinadas como relativamente homogéneas. La Figura 8 indica que, mientras la ciudad se extiende hacia oriente y occidente, la segregación espacial va aumentando hacia las zonas de ladera en el occidente y la margen izquierda del río Cauca en el oriente. En general, pueden considerarse cuatro áreas explicativas del fenómeno, que se diferencian por el valor promedio del índice $(52,1)$. Si el valor del índice es superior o inferior al promedio, este tiende a mostrar mayor o menor grado de segregación. 


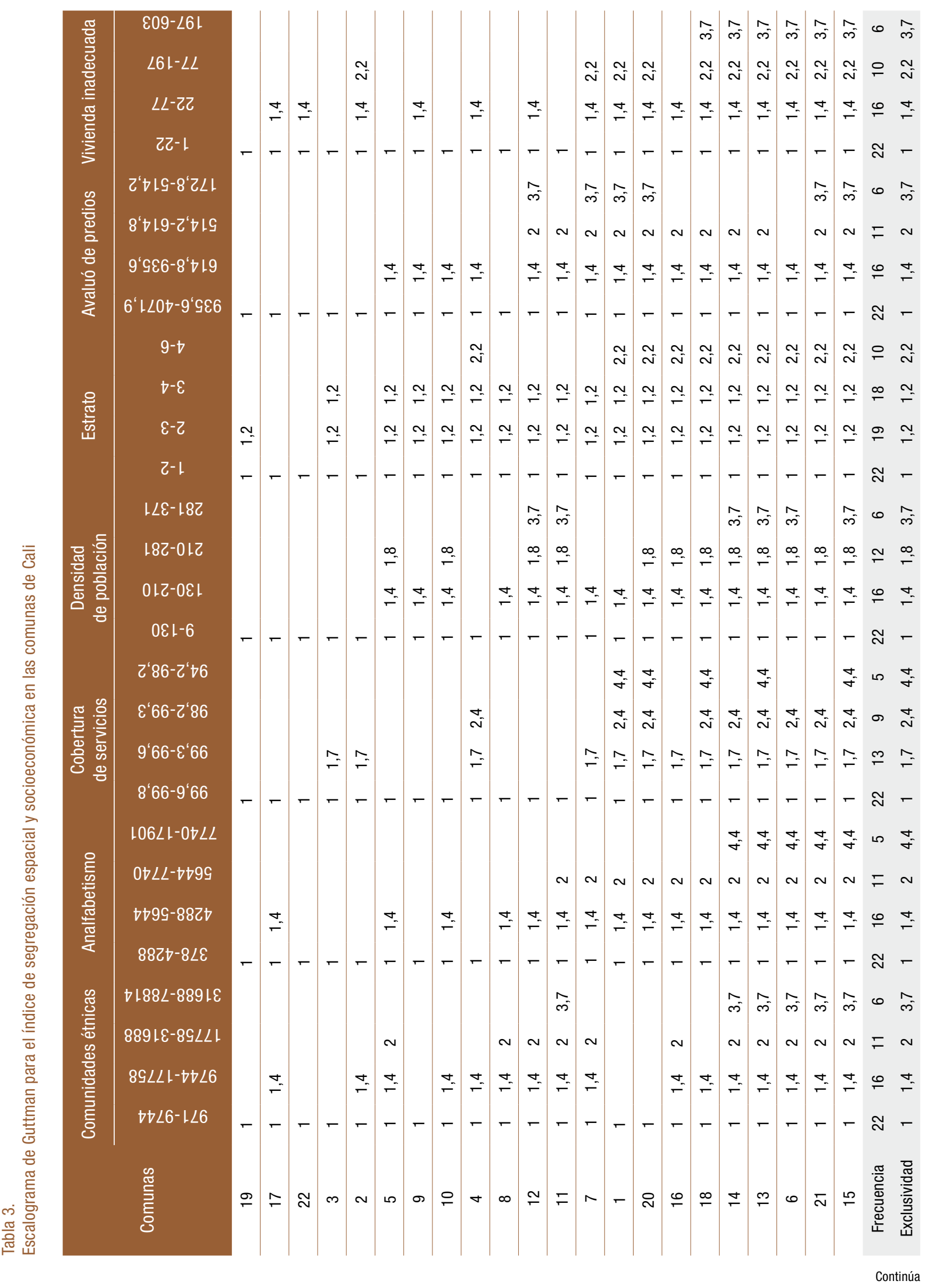




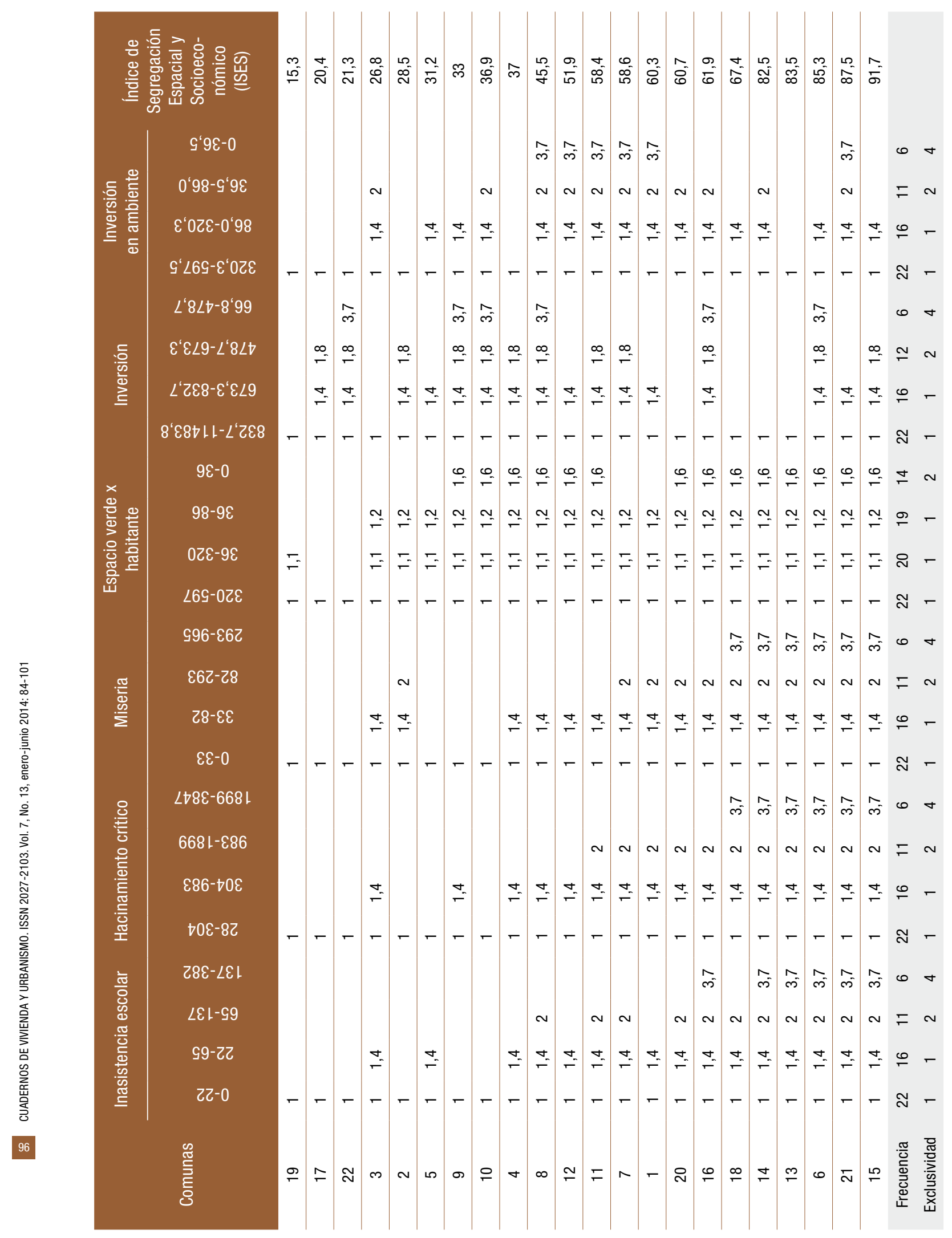

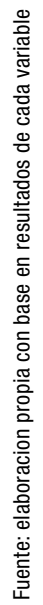


- Zona de segregación espacial y socioeconómica muy alta: conformada por comunas de alojamiento de los grupos pobres y de las comunidades étnicas (afrocolombianos, indígenas, raizales, etc.), sobre todo en la periferia lejana y mal servida; esta zona concentra las comunas del distrito de Aguablanca (13, 14, 15 y 21) ubicadas en el oriente de la ciudad y cuyo desarrollo no ha sido planificado, por lo que presentan los peores indicadores de las variables que componen el índice. Esta zona se caracteriza por hogares en condiciones de miseria, vivienda inadecuada, hacinamiento crítico, índice de vegetación muy bajo, estratos socioeconómicos por debajo de 2 y una cobertura de servicios por debajo de $98 \%$, además de un bajo avalúo de predios, que propicia el asentamiento de inmigrantes y comunidades de bajo poder adquisitivo.

- Zona de segregación espacial y socioeconómica alta: inicia en las comunas cercanas al centro de la ciudad ( 4 y 9) y se extiende hacia el nororiente, sobre las comunas 8 y 12 y al sur, la comuna 10. A ella corresponden comunas de clase económica media a baja, con una presencia significativa de comunidades étnicas y deficiencias en la cobertura de servicios, en las condiciones de la vivienda, baja densidad de vegetación y muy baja inversión del Estado en desarrollo comunitario.

- Zona de segregación espacial y socioeconómica media: corresponde a las comunas de la zona de ladera ( 1 y 20) y dos más en el oriente de la ciudad, sobre el eje vial de la calle 25 (comunas 11 y 16), además de la comuna 7 . Presenta condiciones precarias de los hogares, baja inversión en recuperación ambiental, deficiencia en los sistemas de servicios públicos y una marcada diferenciación social por las condiciones económicas de la zona. No obstante, es importante reconocer que, a pesar de que el avalúo de los predios en la zona es bajo, el asentamiento de comunidades inmigrantes en la zona es bajo, por lo cual sería necesario estudiar otra serie de variables que explique este contraste.
Figura 8.

Índice de segregación espacial y socioeconómica en las comunas de Cali

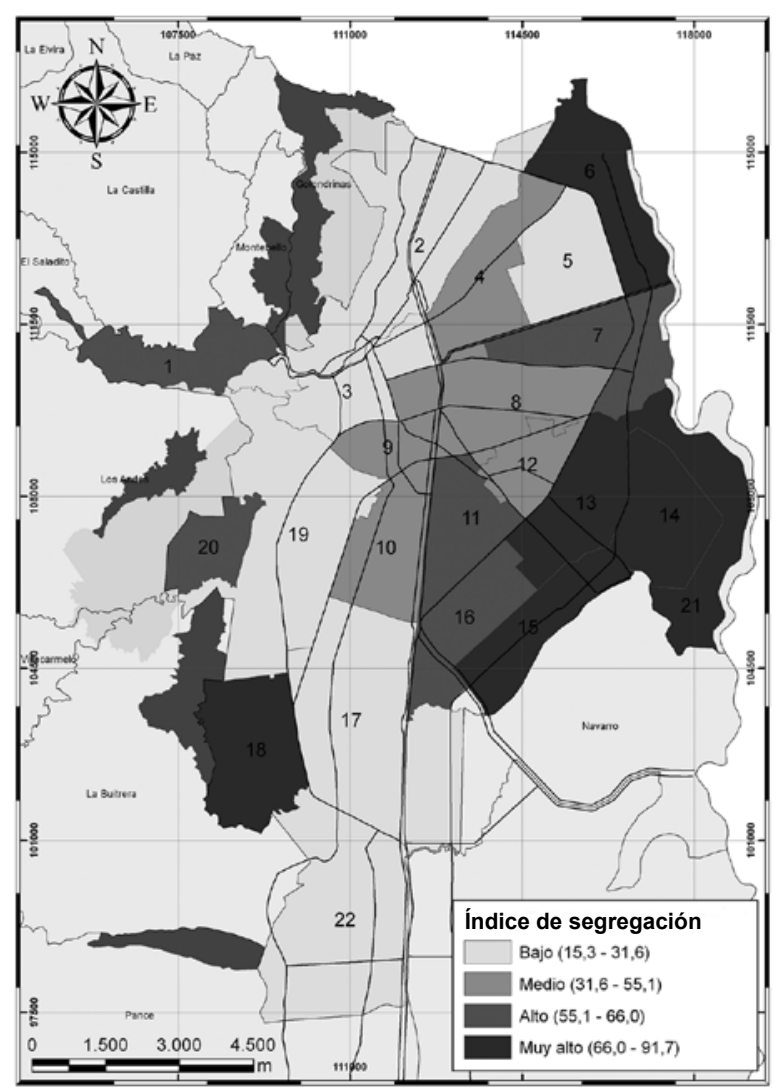

Fuente: elaboracion propia con base en los resultados de la Tabla 3

- Zona de baja segregación espacial y socioeconómica: hace referencia a las comunas localizadas en el occidente de Cali, que forman un eje en sentido sur-norte. Empieza en la comuna 22, se extiende por las comunas 17 y 19 y termina en la comuna 2, al norte de la ciudad. Es importante resaltar que en esta zona debe incluirse la comuna 5, la cual queda cercada por comunas en donde se presenta una segregación espacial de alta a media. Los resultados de esta área contrastan con los del resto de la ciudad, por ser la franja con mejores condiciones ambientales, mayor inversión en ambiente y desarrollo, cobertura de servicios y mejores condiciones de vivienda; además, coincide con las zonas de los estratos socioeconómicos más altos, la menor densidad de 
población y comunas en las que los predios son demasiado costosos para ser adquiridos por población de bajos recursos.

Aunque se hayan determinado zonas homogéneas de segregación espacial y socioeconómica, un análisis detallado de cada una de las comunas, no solo por el orden que ocupan en términos del índice de segregación espacial y socioeconómico, sino del comportamiento de cada variable, permite tomar decisiones con información bastante detallada de las condiciones del sitio, así como de una planificación cuidadosa y diferenciada de las medidas de gestión a implementar, de forma que el impacto social de la inversión pública sea mayor que la generalización de la intervención en toda la ciudad.

\section{Conclusiones}

De acuerdo con los resultados, se puede identificar que la segregación espacial y socioeconómica es un fenómeno complejo. En este trabajo se ha hecho una aproximación general al examen de esta problemática en el contexto urbano y se observa que el grado de complejidad del objeto de estudio es muy elevado, dado que intervienen múltiples factores de diversa índole: socioeconómicos, demográficos, políticos, ambientales y étnicos, entre otros, que a su vez pueden influir de forma directa o indirecta, negativa o positivamente, en los procesos de segregación.

Desde variables estructurales, como las condiciones de vida de la población y las características socioeconómicas, hasta las variables de tipo individual, como la condición de migrante, influyen en el grado de segregación y en la forma en la que los diferentes grupos poblacionales se distribuyen en la ciudad. Por otra parte, existe un debate académico sobre las posibles ventajas y desventajas que supone la segregación, tema que deberá tomarse en cuenta a la hora de disenar y gestionar políticas públicas.
Existe una ciudad para quienes pueden pagarla, con todos los servicios que los avances técnicos permiten, con una sana situación de legalidad y, en la mayoría de nuestras ciudades, en un ambiente que tiene pocos signos de deterioro. La otra ciudad es inundable, sin servicios o equipamientos, sin una seguridad legal, con un entorno ambiental deteriorado. La polarización cada vez mayor que se vive en la sociedad se refleja, así, en la apropiación y construcción del escenario urbano (Clichevsky, 2002, p. 12). La aglomeración de los más vulnerables y la constitución de extensas áreas de pobreza representan el efecto agregado del despliegue de las fuerzas y las motivaciones recién enumeradas. Las élites y los grupos medios van construyendo ciudades a su conveniencia, con la marginación de los beneficios materiales y simbólicos a los más desfavorecidos.

Es importante reconocer que los pocos estudios que se han llevado a cabo sobre segregación espacial y socioeconómica muestran que la exclusión de grupos étnicos tiende a coincidir espacialmente con la segregación de los grupos socioeconómicos de estratos bajos; aparecen concentrados en distintas zonas de la periferia urbana e incluso en zonas centrales muy deterioradas, a diferencia de los grupos socioeconómicos de clases altas que tienden a concentrarse en una sola zona. La uniformidad social del espacio es una de las características más destacadas de la situación de segregación de los grupos socioeconómicos bajos; dicha uniformidad dista de ser un producto de la agregación de las decisiones libres de localización.

\section{Bibliografía}

Ainstein, L. (1998). Globalización, dinámica regional y estructura interna metropolitana: El escenario canadiense. Estudios del Hábitat, (7).

Alvarado, C., Vieyra, A., y Hernández, J. (2008). Diferenciación socio-residencial en el área 
urbana de la ciudad de Cuernavaca, Morelos. Investigaciones Geográficas, (66), 135-152.

Armijo, G. (2000). La urbanización del campo metropolitano de Santiago: crisis y desaparición del hábitat rural. Revista de Urbanismo (3), 1-21.

Arriagada, C., y Rodríguez, J. (2003). Segregación residencial en áreas metropolitanas de América Latina: magnitud, características, evolución e implicaciones de política. En Cepal (editor de la serie), Población y desarrollo: 47. Santiago: Naciones Unidas.

Arriagada, C., y Simioni, D. (2001). Dinámica de valorización del suelo en el área metropolitana del Gran Santiago y desafíos del financiamiento urbano. En Cepal (editor de la serie), Medio ambiente y desarrollo: 44. Santiago: $\mathrm{Na}-$ ciones Unidas.

Barbary, O. (2001). Mesure et réalites de la segmentation socio-raciale à Cali. Une enquête sur les ménages afro-colombiens. Population, 56 (5), 773-810.

Bayona, J. (2007). La segregación residencia de la población extranjera en Barcelona: ¿Una segregación fragmentada? Scripta Nova. Revista electrónica de geografía y ciencias sociales, 11 (235). Recuperado de http://www.ub.edu/geocrit/sn/ sn-235.htm

Brenner, N. (2001). World City Theory, Globalization and the Comparative-Historical Method: Reflections on Janet Abu-Lughod's Interpretation of Contemporary Urban Restructuring. Urban Affairs Review, 37 (1), 124-147.

Buzai, G., Baxendale, C., Rodríguez, L., y Escanes, V. (2003). Distribución y segregación espacial de los extranjeros en la ciudad de Lujan: Un análisis desde la Geografía Cuantitativa. Signos Universitarios, 23 (39), 29-52.
Cabrales, L. F. (coord.) (2002). Latinoamérica: paises abiertos, ciudades cerradas. Guadalajara: Unesco, Universidad de Guadalajara.

Castro Bonaño, M. (2002). Indicadores de desarrollo sostenible urbano: una aplicación para Andalucía. (Tesis doctoral inédita). Universidad de Málaga, España.

Celemín, J. (2012). Asociación espacial entre fragmentación socioeconómica y ambiental en la ciudad de Mar del Plata, Argentina. EURE, 38 (113), 33-51.

Cepal. (2002). Globalización y desarrollo. Recuperado de http://www.eclac.org/publicaciones/ xml/3/10033/sintESPANOL.pdf

Checa, O., y Arjona, G. (2006). Ecología factorial en Roquetas de Mar (Almería). La importancia de las migraciones en la configuración socioespacial. Scripta Nova. Revista electrónica de geografia y ciencias sociales, 10 (219).

Clichevsky, N. (2002). Pobreza y políticas urbano-ambientales en Argentina. En Cepal (editor de la serie), Medio ambiente y desarrollo: 49. Santiago: Naciones Unidas.

De Mattos, C., e Hidalgo, R. (eds.). (2007). Santiago de Chile: movilidad espacial y reconfiguración metropolitana. Santiago: Geolibros.

Departamento Administrativo de Planeación. (2011). Análisis estadistico del Sisbén III en el municipio de Santiago de Cali. Cali: Autor.

Escobar-Morales, G. (comp.) (2012). Cali en cifras 2011. Cali: Departamento Administrativo de Planeación.

Escobar, L. (2008). Indicadores ambientales sintéticos: una aproximación conceptual desde la estadística multivariante. Gestión y Ambiente, 11 (1), 121-140. 
Fullaondo, A. (marzo, 2007). La segregación residencial: valoraciones e indicadores. En Experiencias para el alojamiento y el habitar de la población migrada en Andalucia. Seminario llevado a cabo por la Facultad de Arquitectura de la Universidad de Sevilla, Sevilla.

Fullaondo, A. (2008). Inserción y lógica residencial de la inmigración extranjera en la ciudad: el caso de Barcelona. Scripta Nova. Revista bibliográfica de geografía y ciencias sociales, 13 (795).

Janoschka, M. (2002). El nuevo modelo de la ciudad latinoamericana: Fragmentación y privatización. EURE, 28 (85), 11-29.

Juárez, L. (2006). Segregación urbana y sus implicaciones en las ciudades: una aproximación teórica. Revista de Investigación Cientifica en Arquitectura, 1 (2), 45-50.

Katzman, R., Retamoso, A., y Corbo, G. (2003). La evolución del sistema urbano uruguayo. Subtópico 4: Una aproximación al Fenómeno de la delincuencia y criminalidad en Montevideo. Princeton: Princeton University.

Leal, J. (2007). Cambios en la morfología social de las grandes ciudades españolas: apuntes para una investigación. Arquitectura, Ciudad y Entorno, 1 (3), 150-158.

Lévy, J., y Brun, J. (2002). De la extensión a la renovación metropolitana: mosaico social y movilidad. En F. Dureau, V. Dupont, É. Lelièvre, J. P. Lévy y T. Lulle (coords.), Metrópolis en movimiento: Una comparación internacional (pp. 147161). Bogotá: Alfaomega Colombiana.

Loaiza, W., y Buitrago, O. (2009). Una geografía de los homicidios en Santiago de Cali. (Tesis de pregrado). Universidad del Valle, Colombia.

Martínez, E., y López, A. (2002). El desarrollo de la morfología social y la interpretación de las grandes ciudades. Scripta Nova. Revista electrónica de geografía y ciencias sociales, 6 (112). Recuperado de http://www.ub.edu/geocrit/sn/ sn-112.htm

Martínez, P. (2005). La integración subregional y la caracterización funcional, morfológica del fenómeno de metropolización en el área de influencia metropolitana de Cali. Actas Latinoamericanas de Varsovia, 28, 67-91.

Massey, D., White, M., y Phua, V. (1996). The Dimensions of Segregation Revisited. Sociological Methods \& Research, 24 (2), 172-206.

Meyer, K., y Bahr, J. (2001). Condominios in Greater Santiago de Chile and their impact on the urban structure. Die Erde, 132 (3), 293-321.

Observatorio Internacional de la Democracia Participativa [OIDP]. (julio, 2008). Segregación espacial y territorio en los grandes centros urbanos. Conferencia presentada en el XIV Congresso Brasileiro de Sociologia, Rio de Janeiro, Sociedade Brasileira de Sociologia.

Observatorio Social [OS]. (2007). Visión Cali. Una caracterización para Santiago de Cali. Cali: Autor.

Polanco, C. (2006). Indicadores ambientales y modelos internacionales para toma de decisiones. Gestión y Ambiente, 9 (2), 27-42.

Rodríguez, V. (2001). Segregación residencial socioeconómica: ¿qué es?, ¿cómo se mide?, ¿qué está pasando?, ¿importa? En Cepal (editor de la serie), Población y desarrollo: 16. Santiago: Naciones Unidas.

Rondinelli, D. (1988). Método aplicado de análisis regional. La dimensión espacial de la politica de desarrollo. Medellín: Gobernación de Antioquia, Banco Central Hipotecario. 
Sabatini, F. (septiembre, 1999). Tendencias de la segregación residencial urbana en Latinoamérica: reflexiones a partir del caso de Santiago de Chile. Conferencia presentada en el Seminario Latin America: Democracy, markets and equity at the Threshold of New Millenium, Uppsala, Universidad de Uppsala.

Sabatini, F. (2003). La segregación social del espacio en las ciudades de América Latina. Santiago: Banco Interamericano de Desarrollo, Departamento de Desarrollo Sostenible.

Sabatini, F., Cáceres, G., y Cerda, J. (2001). Segregación residencial en las principales ciudades chilenas: tendencias de las tres últimas décadas y posibles cursos de acción. EURE, 27 (82), 21-42.

Sachs-Jeantet, C. (1995). Ciudad y gestión de las transformaciones sociales. Unesco, Documentos de debate, (2), 1-24.

Sassen, S. (1991). The Global City. New York, London, Tokio. 2da ed. Nueva Jersey: Princeton University Press.

Stallings, B. (diciembre, 2001). Globalization and Liberalization: The Impact on Developing Countries. En Cepal (editor de la serie), Macroeconomia del desarrollo: 4. Santiago: Naciones Unidas.
TAU Consultora Ambiental. (2000). Sistema español de indicadores ambientales: área de medio urbano. Madrid: Centro de publicaciones del Ministerio de Medio Ambiente.

Taylor, P. (2003). Regionality in the World City Network. International Social Science Journal, 56 (181), 361-372.

Universidad San Buenaventura, seccional Cali. (2011). Armonización territorial en la escala subregional sur del valle geográfico. Parte 1: patrones de desarrollo espacial en el entorno metropolitano de Cali. (Informe No. 1). Cali: Autor.

Uribe, O. G. (1998). Geografia y sociedad: exploraciones en compromisos y propuestas actuales. México D. F.: Centro de Investigaciones Científicas.

Vásquez B., E. (2001). Historia de Cali en el siglo 20: sociedad, economía, cultura y espacio. Cali: Universidad del Valle.

White, M. (1983). The Measurement of Spatial Segregation. American Journal of Sociology, 88 (5), 1008-1018.

White, P. (1995). Immigrants and the Social Geography of European Cities. En R. King (ed.), Mass Migration in Europe: The Legacy and Future (pp. 65-82). Londres: Belhaven. 